\title{
Decentralization and Regional Development in Albania
}

\author{
Dorina Klosi (Leka) \\ PhD candidate at European University of Tirana
}

\section{Doi:10.5901/mjss.2013.v4n9p469}

\section{Abstract}

This study aims to discuss the idea of decentralization process of local authorities in Albania and also its impact on territorial administrative organization. Using her experience of several years in audit structures of local government, the author of this study intends to show how much and how has affected the territorial division in the absence of efficiency of public services provided by local authorities. Why the governments (even though the issue of territorial reorganization was their priority) could not do anything in this regard in years? Albania's territorial reorganization, in the current stage when the decentralization reform has entered the third phase of its development is closely related to regional development of the territory. Both (as reorganization and regional development) require the design and implementation of macroeconomic strategies based on detailed analysis of costs and efficiency of public services provided by local governments, harmonizing the instruments in implementing the economic policies of general government (local and central government), but without affecting in any way the principle of decentralization.

II territorio è molto più di una linea sulla mappa, è una caratteristica fondamentale della vita politica e sociale. Esso fornisce il quadro appropriato per l'interazione politica, sociale, rapporti economici e di identità. Per lo Stato moderno, il territorio è importante per due aspetti: rafforza lo stato come principio di dominazione e di controllo - l' autorità e la legittimità dello Stato viene limitata dalla base territoriale; struttura il sistema di rappresentanza e la partecipazione al suo interno. Altrettanto il territorio incoraggia l'interazione economica e riequilibria in modo reciproco la struttura dei mercati, ed è un fattore nella riproduzione della lingua, la cultura, i costumi e la base per l'identità e la solidarietà collettiva.

Uno dei risultati naturali del processo di decentralizzazione in Albania è stata l'organizzazione territoriale del paese.

Dopo il 1991, l'Albania è stata coinvolta nel processo di decentralizzazione della governance in tutti gli aspetti: politico, economico, fiscale e amministrativo . La decentralizzazione in Albania ha incluso tre fasi:

- Nella prima fase 1991-1998 la decentralizzazione politica e il trasferimento di deconcentralizzazione sul decentramento. In questa fase le riforme fiscali ed economiche sono in ritardo con quelle politiche.

- La seconda fase, all' inizio nel 1999 con l'annuncio della Strategia Nazionale per la Decentralizzazione e l'Autonomia Locale e conseguentemente l' adozione della legge $n^{\circ} 8652$ in data 31.07.2000 "Per l'organizzazione e funzionamento del governo locale "

La divisione territoriale dell'Albania si basa sulla legge n. 8652/2000 " Per l'organizzazione e funzionamento del governo locale " e la Legge n. 8653/2000 "Per la divisione amministrativa e territoriale", che hanno portato alla creazione di 373 unità locali di primo livello (65 comuni urbani e 308 comuni rurali) e 12 unità locali di secondo livello, i distretti (Qarku). II Comune della citta' (bashkia) rappresenta una unità territoriale-amministrativa e una comunita di abitanti soprattutto nelle aree urbane e in casi particolari comprende anche le zone rurali. Il territorio, il nome del comune e la sua città centrale sono determinate dalla legge. II Comune del paese rappresenta altrettanto anche una unità territoriale-amministrativa ed una comunità di residenti soprattutto nelle zone rurali e nei casi speciali in aree urbane. II territorio e il nome del comune sono determinati dalla legge. I Distretti rappresentano l'unita' territoriale-amministrativa, che include alcuni comuni delle citta'e paesi, legati geograficamente, tradizionalmente, economicamente, socialmente e hanno interessi comuni tra loro.

La legge definisce chiaramente il ruolo e le responsabilità dei comuni, mentre il ruolo del distretto non è stato determinato in modo chiaro ed è rimasto oggetto per le successive revisioni della legislazione. Attualmente secondo la legge organica questo ruolo consiste nella : progettazione e coordinamento delle politiche e delle strategie a livello regionale, il coordinamento tra il governo centrale e governo locale, offre servizi delegati dal governo centrale alle unità locali che fanno parte del Distretto con la volonta di queste ultime. Ma per molti motivi il Distretto non ha funzionato ed è ancora oggetto di iniziative del governo nel quadro della riforma di decentralizzazione e dello sviluppo regionale. 


\section{Una delle questioni più controverse come detto sopra è stato e rimane tuttora la divisione del territorio .}

La riforma territoriale è stata effettuata nel 2001 sulla base della normativa sopranominata e preceduta dalla riforma di decentralizzazione, ma questo processo non è stato registrato nelle mappe e attualmente i confini attuali sono fonte di conflitti tra unità locali vicine.

Con l'attuale divisione del territorio e la forma di decentralizzazione, l' efficienza e l' economia di scala non vengono raggiunti, per cio' viene leso il principio di sussidiarietà. Possiamo citare il caso del trattamento finale dei rifiuti solidi, la forma del trasferimento di competenze su acquedotti e sistema fognario, la pianificazione del territorio dove devono essere prese in considerazione le implicazioni sulle unità locali vicine, il trasporto pubblico interurbano, le capacità amministrative locali non al livello con la gravita' del problema che devono affrontare, ecc. A causa della mancanza di efficienza e della legislazione sugli standard, si forniscono servizi di qualità scadente e mancanza di accesso, cosa che compromette le finalità di decentralizzazione, che è la fornitura di servizi di qualità per i cittadini.

Secondo una elaborazione statistica dei dati da parte dell'Istituto per lo Sviluppo dell' Abitazione ${ }^{1}$, e' da sottolineare che le informazioni sono scarse e la maggior parte si basano sulla regione (la regione era una forma di organizzazione ereditata dal sistema centralizzato e divide l'Albania in 36 regioni) ancora meno sulle basi delle unità governative locali si osservano queste tendenze di riorganizzazione territoriale:

- La Popolazione in valore assoluto e la densità per $\mathrm{km}^{2}$. circa il $57 \%$ delle unità locali hanno sotto 100 residenti/km², e circa il 28\% hanno densità di 101-300 residenti/km². Mentre circa il 10\% delle unità locali hanno meno di 2.000 residenti e circa il 60\% delle unità locali hanno una popolazione 2.001-10.000 residenti.

- La distribuzione delle forze politiche secondo gli assessori locali e sindaci, mostra una tendenza quasi uguale in tutto il paese. La forza politica della destra domina al centro e nel nord, la forza sinistra nel centro e al sud. Un tale punto di vista sarebbe certamente significativo per ogni partito politico al momento della presa delle decisioni sulla riorganizzazione territoriale. Tuttavia, in molti casi si nota il fatto che l'appartenenza politica del sindaco dell' unita' locale non coincide con la maggior parte dominante del Consiglio, cosa che dimostra che per le elezioni locali si e' perso il legame elettorato- forza politica e rafforza il legame elettorato-candidato politico.

- II fattore storico è diventato un confronto tra la mappa dei distretti nel 2008 e delle prefetture nel 1930 e della mappa delle regioni nel 2008 e delle sottoprefetture del 1930 e si e' scoperto che le regioni omogenee dal punto di vista culturale sono divise in tre o più parti nei distretti di oggi, oppure regioni diverse dal punto di vista culturale sono riunite in un unica regione.

- Le mappe degli indicatori di povertà secondo I' INSTAT ${ }^{1}$, visualizzano regioni virtuali le quali nel caso di indicatori di povertà e della povertà estrema hanno la stessa tendenza, e nel caso di disuguaglianza delle ragioni mostra un altro andamento. Cosi nel primo caso si nota molto la divisione nord / centro / sud, e nel secondo caso si creano delle regioni nord-ovest / nord-est / centro / sud.

Tutte le mappe degli indicatori finanziari, delle proprietà pubbliche e dei servizi sociali dividono in modo visibile la pianura occidentale dalle zone di montagna e dal sud-est. La pianura occidentale ha delle risorse umane ed economiche e delle opportunità di generare reddito alto . Altrettanto l'efficienza in termini di rapporto risorse / abitanti e' più elevata nel corridoio Tirana-Durazzo ulteriormente nella pianura occidentale e molto meno nelle altre regioni dell'Albania.

"Nessuna comunità, compresa la Comunità europea, non può essere stabile, se il tenore di vita delle persone che la compongono non viene descritto come non I' uguaglianza tra gli individui, e se esiste anche il piu' piccolo dubbio sulla volontà degli Stati membri di questa comunità per aiutare gli omologhi "1

Per raggiungere il grande obiettivo molto desiderato per l' integrazione nella UE, l'Albania deve rispettare gli accordi e adempiere agli obblighi previsti e con i documenti firmati. La regionalizzazione 0 una forma di regionalizzazione non è obbligatoria, ma le capacita' istituzionali e umane per consentire l' acquisizione e la gestione dei fondi dell' IPA, sono obbligatori. Secondo le politiche di sviluppo regionale dell' UE si raccomanda che lo sviluppo regionale non deve essere basato su una politica di distribuzione, ma nel uso dei vantaggi competitivi della regione. Per quanto sopra, non e' obligatoria la regionalizzazione, la divisione territoriale, ma se l'obiettivo è l'integrazione nella UE, questi problemi non possono essere evitati.

Nel 2007 il Ministero dell'Economia ha preparato la Strategia Intersettoriale per lo Sviluppo Regionale (SNZHR), documento che e' nato a causa della crescente difficoltà del Governo albanese per lo sviluppo economico e sociale

${ }^{1}$ EU 2004, Le Politiche Regionali 
sempre più diseguale, tra le diverse parti del territorio e aveva come scopo: fornire un approccio coordinato per un sostenibile sviluppo socio-economico di tutte le regioni del paese, e la creazione di legami tra le istituzioni di qualsiasi livello di governo e i gruppi di interesse per lo sviluppo sostenibile e per la riduzione delle disuguaglianze socioeconomiche.

Nell' SNZHR e' previsto lo sviluppo di due programmi di investimento: II Programma Nazionale per lo Sviluppo dei Distretti e il Programma di Sviluppo delle Zone Svantaggiate. A livello istituzionale SNZHR, prevede la costruzione di un Agenzia Nazionale per lo Sviluppo Regionale insieme a 12 Agenzie di Sviluppo Regionale, per ogni uno dei distretti, la costruzione di 12 Consigli di Partenariato del Distretto e il Consiglio di Partenariato Nazionale per lo Sviluppo Regionale. Altrettanto SNZHR propone anche lo sviluppo di 12 strategie di sviluppo a livello di Distretto , così come una legge sullo sviluppo e la regolamentazione regionale per la sua attuazione.

Secondo l' SNZHR la disparità regionale in Albania è presente in forme estreme. Così:

- La povertà si presenta del $66 \%$ più alta nelle zone di montagna che a Tirana e il $50 \%$ più alta che negli altri grandi centri urbani.

- Tirana ha un indice del PIL di 0,772 a fronte di un indice di PIL di 0,252 di una quota delle zone montane e di un indice di sviluppo umano di 0,830 a fronte di un indice di 0,632 delle zone montane.

- II livello di disoccupazione a Kukes è 3 volte superiore a quello di Tirana e la povertà 2 volte superiore rispetto a Valona. La popolazione di Valona ha 2 volte più accesso ad acqua corrente di quelli in Dibra. I residenti di Tirana hanno 2,5 volte in più accesso a visite mediche di quelli a Kukes.

- La migrazione interna risulta con enormi disparità interne. Negli anni 2005-2006 la popolazione di Tirana è aumentata di 137.000 persone e quella di Durazzo di 43.000 persone. Mentre la popolazione di Dibra si è ridotta di 43.000 persone (una riduzione del $23 \%$ della popolazione del distretto) e quella di Kukes di 30.000 persone (una riduzione del $27 \%$ della popolazione del distretto) ${ }^{2}$

Secondo SNZHR I'Albania ha un serio problema nell' approfondimendo della disuguaglianza . ${ }^{3}$

Tab.1

\begin{tabular}{|c|c|c|c|c|c|c|c|c|}
\hline $\mathrm{Nr}$ & Distretto & Popolazione & $\begin{array}{c}\text { Livello di } \\
\text { poverta / media } \\
\text { nazionale. } \\
\%\end{array}$ & $\begin{array}{c}\text { Livello di } \\
\text { disoccupazione } \\
\text { Imedia } \\
\text { nazionale } \\
\%\end{array}$ & $\begin{array}{c}\text { Reddito } \\
\text { locale/ } \\
\text { Media } \\
\text { Nazionale } \\
\%\end{array}$ & $\begin{array}{c}\text { Accesso } \\
\text { fornimento } \\
\text { acqua /Media } \\
\text { nazionale } \\
\%\end{array}$ & $\begin{array}{c}\text { Nr. Visite } \\
\text { mediche / } \\
\text { Media } \\
\text { nazionale } \\
\%\end{array}$ & $\begin{array}{c}\text { Licenza } \\
\text { scuole } \\
\text { d'obbligo/Me } \\
\text { dia nazionale } \\
\%\end{array}$ \\
\hline 1 & Berat & 181901 & 96 & 99 & 74.6 & 114 & 107 & 368 \\
\hline 2 & Diber & 166367 & 59 & 85 & 61.4 & 54 & 70 & 55 \\
\hline 3 & Durres & 280996 & 102 & 117 & 106 & 131 & 68 & 53 \\
\hline 4 & Elbasan & 380593 & 80 & 110 & 70 & 83 & 62 & 147 \\
\hline 5 & Fier & 380737 & 85 & 133 & 77 & 91 & 83 & 76 \\
\hline 6 & Gjirokaster & 80646 & 131 & 118 & 112 & 97 & 132 & 385 \\
\hline 7 & Korce & 263586 & 94 & 126 & 78 & 97 & 127 & 202 \\
\hline 8 & Kukes & 102036 & 63 & 42 & 74 & 91 & 56 & 54 \\
\hline 9 & Lezhe & 159882 & 69 & 61 & 78 & 58 & 89 & 162 \\
\hline 10 & Shkoder & 250351 & 77 & 52 & 67 & 74 & 120 & 75 \\
\hline 11 & Tirane & 677871 & 108 & 168 & 160 & 123 & 140 & \\
\hline 12 & Vlore & 202295 & 139 & 89 & 139 & 132 & 92 & 506 \\
\hline & & 3127261 & & & & & & \\
\hline
\end{tabular}

In tutti i documenti proposti dalla SNZHR l'attenzione si concentra al Distretto e si cerca di formulare il suo ruolo di catalizzatore e coordinatore dello sviluppo regionale. Quando il Distretto in primis non è stato concepito nella sua creazione come la Regione, anche se puo' essere addattabile, e inoltre le istituzioni responsabili per lo sviluppo regionale che sono previste per lavorare a livello regionale possono funzionare anche se il Distretto non esiste.

Daltra parte i Distretti durante questo periodo, hanno visto in modo vago le loro funzioni e non sono mai riusciti precisamente a svolgere un ruolo di coordinamento delle politiche tra il governo locale ed il governo centrale, e spesso hanno avuto un ruolo dominante sul ruolo delle Prefetture, le quali sono le istituzioni che eseguono il deconcentramento

${ }^{2}$ Fonte Documentazione SNZHR , on line

${ }^{3}$ Le scifre della tabella sono prelevate de datto del INSTAT, MB, MPCSSHB, MASH, MSH, MPPTT, Documentazione SNZHR online 
del potere in Albania. La stessa amministrazione e i Consigli dei Distretti non sentono la responsabilita' necessaria verso i cittadini, influenzati anche dal fatto che essi non sono eletti direttamente dal popolo ma nominati.

Dal momento in cui SNZHR e' stato progettato, realmente non è stato applicato. Nell'agosto del 2008 è stato sviluppato il progetto di legge per lo sviluppo regionale, che non è ancora presentato al Consiglio dei Ministri 0 al Parlamento. L'unico strumento che indirizza fino ad un certo punto il problema di sviluppo regionale è il Fondo per lo Sviluppo delle Regioni (FSR), che è uno strumento finanziario finalizzato all' allocazione di fondi centrali per gli investimenti nelle unità locali su base competitiva ed ha per obiettivo l' armonizzazione delle politiche del bilancio nazionale e locale. A questo proposito vale la pena sottolineare alcune questioni come:

- I fondi FSR vengono distribuiti sulla base di criteri definiti con atto normativo ${ }^{4}$, ma le concessioni dei finanziamenti competitivi le rendono più orientate verso i progetti che rispondono allo sviluppo regionale. In realtà, questa logica è in linea con quella della Legge nr. data "Sulla gestione del bilancio nella Repubblica di Albania", entrata in vigore nel gennaio 2008 e lavora in conformità con la logica dei programmi e dei progetti.

- La natura competitiva crea il problema del "distretto chiuso a bassa capacità" (cosa che ha avuto luogo in Bulgaria), il che significa opportunità di sviluppo solo per un gruppo limitato di partner, di solito quelli piu' sviluppati. Così il debole rischia di indebolirsi ancora di più, se a lui non è offerto un sostegno particolare. ${ }^{5}$

- C'è poca conoscenza su FSR e la modalità di applicazione e gran parte delle unita locali operano con il vecchio formato delle gare competitive. Questa conclusione è emersa da un sondaggio effettuato da un progetto di UNDP.

- E infine, per tutte le istituzioni che verrano coinvolte nella gestione di questo fondo si ha bisogno di rafforzare le capacità esistenti e la costruzione di nuove capacità.

Ci sono due obiettivi fondamentali su cui si basa la Politica Europea di Coesione: l'efficienza e l'equità 6 . Mentre SNSR enfatizza principalmente lo sviluppo del quadro giuridico e le capacità istituzionali e non risponde alla domanda se in un paese come l'Albania si lottera' per l'efficienza o l'equità? La risposta a questa domanda certamente influenzera' la divisione territoriale, l'efficienza e ruolo dei distretti, la creazione delle regioni, nella loro forma e dimensione.

Secondo I' SNSR l'approccio allo sviluppo regionale è più mirato all' interno piuttosto che integrato con il concetto europeo di sviluppo regionale (RD).

Ma i fondi che l'Albania beneficerà dall' IPA III varrano gestiti a livello centrale, si concentrerano inizialmente sulle priorità dell' UE e le priorità nazionali, attraverso tanti grandi investimenti infrastrutturali per i trasporti, l'ambiente e la competitività regionale.

II bilancio IPA III finanzierà progetti con valori molto grandi (valori che supereràno i 10 milioni di Euro), questo significa che al primo momento l'Albania non sarà in grado di affrontare i suoi problemi regionali attraverso questi fondi. Come inizio, e interessante di trattare le disuguaglianze tra il nostro paese come singola regione dell'UE e di altri paesi della Comunità.

Pertanto ciò che si richiede è una convergenza dello Sviluppo Regionale interno con IPA III e un graduale adattamento del Fondo per lo Sviluppo Regionale come unico strumento attuale e concreto per questa convergenza.

In termini di risorse limitate di tempo, denaro e umano questa alternativa è un occasione per essere più efficienti con le risorse utilizzate sensa rischiare di perdere l' orientamento strategico.

Per realizzarlo con successo si richiede:

- Cambiamenti limitati nella struttura istituzionale che permettono di concentrarsi maggiormente sullo sviluppo delle capacità umane che dovrebbero beneficiare di conoscenze ed esperienze.

- Mantenere le responsabilità gestionali e di programmazione a livello centrale e la specializzazione delle unità locali nella gestione dei progetti.

- Una chiara definizione delle competenze nella realizzazione degli investimenti tra le autorita' locali e centrali, al fine di essere in grado di evitare potenziali conflitti di finanziamento, la gestione e la programmazione.

- Inoltre, essa richiederebbe alcune modifiche che si possono fare al Fondo di Sviluppo Regionale: Rivedere la base dove si costruisce per spostarlo dall' attuale attenzione verso i progetti a favore di programmi di sviluppo. Ciò consentirà una migliore connessione del FSR con la legislazione di bilancio.

\footnotetext{
${ }^{4}$ Decisione del Consiglio dei Ministri nr. 135, date 3.02.2010 "Per la detterminazione dei critteri per la somminsitrazione dei fondi per 0 sviluppo delle regioni."

${ }^{5}$ Marinov, 2010

${ }^{6}$ Barca 2009
} 
Collegamento migliore del FSR che viene gestito centralmente con le strategie di sviluppo dei distretti speciali.

- Assistenza particolare per le aree svantaggiate.

In conclusione, in ogni caso tutto dipende dai progressi che l' Albania farà negli sforzi per la creazione di un quadro istituzionale e delle capacità umane, che devono essere essere esperti in gestione, in programmazione $\mathrm{e}$ attuazione del sostegno finanziario dell' UE, con l'obiettivo finale di fornire i fondi ed un loro uso efficiente .

\section{Reference}

Barca F, 2009 Un'agenda per una politica di coesione riformata, www.eurada.org/site/files/regional1\%20development/Barca_report.pdf Co-PLAN, dicembre 2008, Istituto per lo Sviluppo degli Habitat, Documento delle politiche l'impatto delle Politiche decisionali Commissione Europea, 2004, Report di Progress su Albania

Decisione del Consiglio dei Ministri nr. 135, date 3.02.2010 " Per la detterminazione dei critteri per la somminsitrazione dei fondi per 0 sviluppo delle regioni ."

Fonte Documentazione SNZHR, on line

Le scifre della tabella sono prelevate de datto del INSTAT, MB, MPCSSHB, MASH, MSH, MPPTT, Documentazione SNZHR online Legislazione per il governo locale, on line www.qpz.gov.al

Marinov V, D. Malhassian 2002, Misure e valutazioni di disparità regionali e le loro implicazioni politiche: il caso della Bulgaria

McClements C, 2010, Cros-cutting strategy for regional development, Albania, Review

OECD, 2009, Come si sviluppano le regioni, www. oecd.org/pubblicazioni/policybriefs

Strategia Intersettoriale per lo Sviluppo Regionale, 2007 on line 